\title{
XXVIth ASTIN COLLOQUIUM \\ Leuven, Belgium, 1995
}

The 26th ASTIN Colloquium was held at the "Hoge Heuvel" college in Leuven from 17 to 19 September 1995. It has been organized under the auspices of the ARAB-KVBA (Association Royale des Actuaires Belges - Koninklijke Vereniging van Belgische Actuarissen). Due to the centennial celebration of the International Association of Actuaries, it took place in conjunction with the 25th International Congress of Actuaries, which was organized in Brussels the week before.

Over 150 actuaries and 30 accompanying persons from more than 20 countries attended.

The colloquium started informally on Sunday 17 September with the registration of the guests at Hotel Holiday Inn and a reception in the Promotion Hall of the Catholic University of Leuven. The well-known Belgian beer served at this welcoming reception provided an atmosphere of conviviality, which was to be maintained during the following days.

At least part of the participants of the colloquium had to get up very early on Monday, because their companions left Leuven for Amsterdam at 7 o'clock in the morning.

The opening ceremony on Monday started the scientific programme. Welcoming addresses were delivered by R. De Bondt (Dean of the Faculty of Economics and Applied Economics of the Catholic University of Leuven), J.M. Maes (ARABKVBA) and M. Goovaerts (chairman of the scientific committee of the colloquium).

The first invited lecture, immediately after the opening ceremony was given by J. Beirlant and J. Teugels on the topic Modelling Large Claims in Non-Life Insurance. Chairmen were R. Norberg and M. de Lourdes Centeno.

The rest of the morning and part of the afternoon were devoted to working sessions on topic number 1 Statistical Methods and IBNR Techniques under the chairmanship of H. Panjer and E. Pitacco. A survey of the 9 papers presented on this topic was given by J.M. Rheinhard.

The subsequent session dealt with colloquium topic number 2 Models for Life Insurance. Chairmen were H. Wolthuis and N. De Pril. J. Dhaene reported on the 3 submitted papers.

In the evening the participants were given the opportunity to make a guided tour of Leuven and to learn something about its history reflected in many historic buildings. The evening ended with a reception in the City Hall, offered by the mayor of Leuven.

On Tuesday, the accompanying persons made a day-trip to Antwerp, a town loved by most women (Diamonds are a girl's best friend).

ASTIN BULLETIN, Vol. 26, No. 1, 1996, pp. 5-14 
That day's scientific programme started with the second invited lecture on Communicating Educational Topics and Research Results by A. Shapiro. Chairmen were B. Jewell and C. Stoop.

The colloquium's third topic on Ruin and Related Topics was chaired by R. Kaas and H. Gerber. W. Van Thielt reported on the 5 presented papers.

The "Speakers Corner" provided an opportunity for anyone wanting to make a short contribution on the topic of his choice. Papers were presented and discussed which had been submitted only shortly before the beginning of the colloquium and could therefore not be sent to the participants in advance. G. Taylor and C. Levy moderated this well-established feature of the ASTIN Colloquia.

After the final working session there was a brief closing ceremony.

In the evening, the traditional colloquium dinner was served in the Faculty Club. The after-dinner speeches were delivered before dinner. Marc Goovaerts, being in a poetic mood, summarized the colloquium in the following poem:

In the past Bob and Bob for Astin wrote an interesting limerick

Dutch actuaries now give solutions that are numeric

While Belgians like Etienne were more interested in geometry

It is left to me to try to construct for you some poetry

In September 95 an Astin colloquium was organized

and Leuven was the place where everything was centralized

The housing was not always that very luxurious

but the service of Marleen and Els was marvellous

More than thirty talks were presented

and all of them were very well attended

Parallel sessions had not to be organized

The multimedia items were sometimes fairly specialized

Several methods were presented that had good qualities

but some others still suffer from unexpected instabilities

In a lot of cases convergence was certified

but first a number of conditions had to be verified

The speakers came from many different nations

and spoke English in all kinds of variations

They gave talks on problems that were classical and new

but completely solved were only a few

There were talks we could easily understand

but some others seemed to have no end

Some speakers used arguments that were sophisticated and deep

but, as you might not believe, nobody was falling into sleep

More actuarially orientated was Hans Bühlmann's intervention in which he gathered data about the participants' number of participations in the other ASTIN Colloquia. The honorary chairman of ASTIN thanked the Belgian organizing committee under the chairmanship of $M$. Goovaerts, for arranging this colloquium in Leuven. The 
1995 ASTIN Colloquium had come to an and. We are looking forward to meeting again in Denmark next year.

Jan Dhaene

\section{APPENDIX : LIST AND SUMMARY OF INVITED LECTURES AND PAPERS}

The following is a brief overview of the contents of the invited lectures and papers that were presented. The summaries of the papers on Topics 1,2 and 3 are the summaries presented by the "rapporteurs" during the working sessions.

\section{Invited lectures}

\section{J. Beirlant and J. Teugels: Modelling Large Claims in Non-Life Insurance.}

In this lecture the basis statistical tools to model the ultimate right tail of a loss distribution were introduced, namely quantile plots and mean excess plots. The slope of a quantile plot describes the spread of the distribution. A plausible tail model can be identified through the ultimate linearity in the right end part of the corresponding quantile plot. The visual impression should be confirmed by mean excess plots which constitute derivative plots of the quantile plots: ultimate constant mean excess plots hence confirm the graphical impression from the quantile plot.

These graphs also enable the estimation of important tail indices, such as the Pareto index. In case of an ultimate linear Pareto quantile plot the derivative plot yields the so-called Hill estimates for the Pareto index. Adaptive estimation of an optimal number of extremes to be used in the estimation procedure can be based on the concept of best linear regression fit through the quantile plot.

The authors emphasized the link between these statistical tools and basic actuarial notions in the framework of excess-of-loss reinsurance: for example, the mean excess value provide estimates of the expected reinsurance amount per claim in such contracts.

The lecture ended with a demonstration from a software implementation illustrating applications to tail index and high quantile estimation.

\section{A. Shapiro: Communicating Educational Topics and Research Results.}

The purpose of this presentation was to demonstrate how interactive computer assisted instruction (CAI) can be used to improve the learning experience of actuarial students and practitioners and to help researchers communicate their ideas and research results. The underlying theme of the presentation was that any idea that can be conceptualized can be presented dynamically on the computer screen.

The presentation began with a description and illustration of the nature of a computer-based multimedia environment. It was computer-based inasmuch as it 
used interactive software to randomly access topics and to dynamically present ideas. It involved multimedia in the sense that it incorporated sounds and videos into the presentation. To emphasize that this could be done at relatively low cost to the user, the entire presentation was conducted using only a $486 \mathrm{SL} 33 \mathrm{Mhz}$ notebook computer.

Next, the presentation demonstrated user-friendly interactive time lines, graphs, tables, simulation, and what-if analysis, and illustrated the use of voice-over and full motion video.

The presentation ended with the conclusion that this technology will be an integral part of actuarial communication in the future.

\section{Topic 1 : Statistical Methods and IBNR Techniques}

\section{A. Parisi and S. Martignolles: Utilisation de Modèles sur les Montants Cumulés} pour Déterminer la loi de Probabilité des Provisions pour Sinistres à Payer en Assurance IARD.

The authors propose to approximate the distribution of the unpaid losses for a given occurrence year by the normal approximation, the Normal Power approximation or the gamma approximation. These approximations permit the construction of a confidence interval for the unpaid losses in order to check the reserves set up by the loss adjusters of the company.

\section{A. E. Renshaw: Claims Reserving by Joint Modelling.}

Arthur Renshaw develops a method based on two joint generalized linear models to estimate the claims reserve. The structure of these models is obtained from assumptions about the incremental claims severities expressed as a function of the delay. The first GLM is used to predict the mean incremental claims, the second to model the variances of these incremental claims which appear as weights in the first model. Starting with a set of initial values for these variances, the two models are fitted alternatively, the convergence of this two stage procedure being monitored by the deviances of the GLM's at each fitting.

\section{O. Hesselager: Modelling of Discretized Loss Reserving Data.}

The papers show first that when the claim process is an inhomogeneous Poisson process and when the data are discretized in the usual way (occurrence year/ reporting year), the distribution of the delay of reporting will in general depend on the occurrence year. This dependence disappears when the seasonal variation in the exposure is the same for each occurrence year. The author analyses the random fluctuations of the delay probabilities when the Poisson parameter is itself a stochastic process satisfying given conditions and proposes credibility estimators taking these fluctuations into account. 


\section{W. Hürlimann: Actuarial Statistics I: Likelihood Characterizations.}

In this paper, classes of "maximum likelihood mean equivalent estimators" are defined. A likelihood estimator asymptotically equal to the maximum likelihood estimator is constructed for Bernoulli mixed discrete-continuous distributions. A characterization of unbiased pseudo-estimators related to the class of maximum likelihood mean equivalent estimators under an orthogonal condition of the parameter of interest is derived and applied to some examples.

\section{W. Hürlimann: Actuarial Statistics II: Exponential Characterizations.}

The well-known characterization of regular exponential families by minimum variance unbiased estimators is considered in this paper. It is shown that the class of regular exponential families with diagonal $2 \times 2$ Bhattacharya matrix and quadratic variance of the observation can be generated from a single elementary distribution (Dirac's distribution or Gauss's distribution for example) using commutative transformations. Some distributions of interest in actuarial mathematics belong to this class.

M. De Lourdes Centeno: Excess of Loss Reinsurance and the Probability of Ruin in Finite Horizon.

The author shows how to choose the retention limit in an excess of loss treaty in order to minimize a known upper bound (Gerber, 1979) for the finite time ruin probability. The author compares Lundberg's upper bound, Gerber's upper bound and the exact ruin probabilities as functions of the retention level in two special cases (exponentially distributed claims and Pareto distributed claims).

\section{J.A. Nelder and R.J. Verrall: Credibility Theory and Generalized Linear Models.}

J. A. Nelder and R.J. Verrall show how credibility theory for exponential families can be incorporated into the general framework of generalized linear models. Within this framework it is possible to widen the range of models which may be useful for actuarial applications.

\section{R.J. Verrall: Claims Reserving and Generalized Additive Models.}

The author demonstrates how the non parametric smoothing techniques allowed by generalized additive models may be applied in claims reserving models. The paper concentrates on the chain ladder technique. It is shown how generalized linear models and generalized additive models for claims reserving may be implemented in the statistical package S-PLUS. The methods are illustrated on a set of data discussed in previous papers. Approximations of the root mean square errors are provided for the predicted values. 


\section{Dannenburg: An Autoregressive Credibility IBNR Model.}

An autoregressive model is considered for IBNR reserves: in this model the payments in one development year are equal to a fixed fraction of the payments in the preceding development year plus a random deviation. Credibility predictors are constructed and estimators of the parameters of the model are provided. Also an extension of the model to the case of a random autoregressive coefficient is presented.

\section{Topic 2: Models for Life Insurance}

R. Norberg: Stochastic Calculus in Actuarial Science: Itô's Revolution - Our Revelation.

The paper demonstrates by examples the usefulness of modern stochastic calculus not only in life insurance, but also for computing total claim distributions in non-life insurance, the probability of ruin in actuarial risk theory and other functionals of practical interest. The tools that are needed from stochastic calculus include the Doob-Meyer decomposition theorem and some other results from martingale theory. An advantage of this approach is that it makes a methodological unification possible as the same set of tools is used for solving several problems from traditionally seggregated subfields of actuarial mathematics.

A. De Schepper, M.J. Goovaerts and R. Kaas: A Recursive Scheme for Perpetuities with Random Positive Interest Rates. Part I. Analytical Results.

In former papers, the authors investigated the probabilistic behaviour of annuities certain when the force of interest is modelled by means of a stochastic process that leaves the possibility of a cumulative force of interest that becomes negative. If the interest should be interpreted as the future return on risky investments, such models should not cause any problem. However, there are situations for which such negative cumulative force of interest has to be avoided. The authors offer a solution to this extended problem, at least for perpetuities. The method requires the fundamental solution of a second order differential equation, which is similar to the well-known Schödinger equation in quantum mechanics.

\section{L. Posey and A. F. Shapiro: Choosing Actuarial Assumptions for Pension Plan Valuations: An Expected Utility Model.}

The authors consider the valuation of a defined benefit pension plan in order to compute the appropriate current years' contribution. Obviously, actuarial assumptions play an important role in this process. However, many of the criteria underlying the choice of the assumptions may be in conflict with one another. For instance, due to concerns about solvency, the actuary has an incentive to choose a contribution which is rather high. But Government officials may interprete this 
behaviour as an attempt to avoid current taxation. This exerts pressure on the actuary to choose a lower contribution than might otherwise be chosen. The authors use expected utility theory to develop a simple model that can be used to determine the appropriate actuarial assumptions when faced with potentially conflicting criteria.

\section{Topic 3: Ruin and Related Topics}

\section{J.-M. Rheinhard: Recursive Evaluation of Ruin Probabilities in the Classical Risk Model.}

The author first recalls an already known recursive procedure for calculating the ultimate ruin and the finite time ruin probabilities in the classical discrete model. He shows that the same scheme can be used to calculate exactly the ultimate ruin and the finite time ruin probabilities in the classical Poisson model with arithmetically distributed claim sizes. In the next section of his paper the author considers the same classical Poisson model but with an arbitrary claims size distribution and uses his previous results to approximate the ruin probabilities by discretizing this claims size distribution. An upper bound is provided for the approximation error and the paper is concluded with numerical illustrations for two particular claims size distributions: a Pareto distribution and a combination of two exponential distributions.

\section{S. Wang: Risk Loads in Life/Non-Life Insurance: A Unified Approach.}

In this paper the author gives a unified approach to risk loads in both life and non-life insurance. This important topic is traditionally dealt with differently by life and non-life insurers who use totally different methods to impose risk loads. To do this the author uses the so-called " $\mathrm{PH}$ (or proportional hazard)-transform premium principle". He recalls its definition and some of its properties, applies it to price a n-year term life insurance and compares the resulting risk load to the one obtained by adding a percentage to the mortality rates as is traditionally done by life insurers.

\section{W. Hürlimann: A Stop-Loss Ordered Extremal Distribution and Some of Its Applications.}

The author describes a two-point distribution, which maximizes the net stop-loss premium of a reinsured risk given the mean, variance and range of the original risk. From that result he derives a continuous extremal distribution, defined on the same range, that gives rise to the same net stop-loss premiums. He derives a recursive formula to calculate all order moments in the case of a finite range and gives explicit formulas for the mean, variance and skewness of that extremal distribution. Finally the author considers several problems like: the maximum likelihood estimation of the parameters of the extremal distribution and its application to a practical example and the calculation of the stop-loss distance to the extremal distribution. 


\section{W. Hürlimann: On Quasi-Mean Value Premium Principles.}

In this paper the author defines a generalized notion of mean value principle, called quasi-mean value principle and considers an important subclass called endogenous quasi-mean value premium principle. He shows that most of the important principles studied today in actuarial science can be considered as special cases of the quasi-mean value principle in general or of the endogenous subclass in particular. In combining the quasi-mean value principle with his own reinsurance based premium principles, he allows for the construction of the so-called generalized Swiss family. The author also studies some fundamental properties of the quasi-mean value premium principle and especially of the endogenous subclass.

\section{H. Schmitter: An Upper Limit of the Stop-Loss Variance.}

The author shows that a stop-loss reinsurer, wanting to calculate the variance of the annual result on a stop-loss cover he writes, can be sure that this variance has an upper limit equal to the variance of a particular two-point distribution, given that the expected value and variance of the original risks as well as the stop-loss risk premium are known. First the author deduces the upper limit of the stop-loss variance when the underlying risk is distributed according to a two-point distribution. Then he shows that this result is also an upper limit when the underlying risk follows any arbitrary distribution.

\section{Speakers Corner}

\section{E. Kremer: Credibility for Stationarity.}

In this paper results for the linear credibility estimators are given for general stationary premium models. The results are derived by applying recursions and formulas of the prediction theory of stationary stochastic processes. The paper extends former results for ARMA-processes.

E. Vandermeulen and R. Detry: Mesure du Risque d'un Excédent de Sinistre en cas de Convergence de la Distribution du Coût des Sinistres vers une Loi de Pareto.

The authors consider a portfolio consisting of a fixed number of compound Poisson distributed random variables with Pareto distributed claim accounts. The adjustment coefficient is determined before and after reinsurance of this portfolio.

\section{R. Kaas and O. Hesselager: Ordering Claim Size Distributions and Mixed Poisson Probabilities.}

The authors investigate various orderings between continuous distributions for severities, having the same first $n$ moments. General results are derived which 
establish an ordering between such distributions, and these results are applied to compare the Gamma, the Inverse Gaussian and the lognormal distributions with equal means and variances. Finally, the situation where such continuous distributions are used as mixing distributions in mixed Poisson models for claim numbers, is considered. It is shown that the order properties of the mixing distributions are inherited by the corresponding mixed Poisson distributions.

\section{Picech and L. Sigalotti: Equilibrium Premiums and Some Efficiency Measures for Bonus-Malus Systems.}

The authors consider the predictive accuracy of a Bonus-Malus (BM) system, which assesses the efficiency by using a mean over time of the expected square differences between BM-premiums and expected claims. They also introduce a measure of the mutuality implied by a BM-system. They consider three indexes of efficiency by which it is possible to investigate the capability of the system to discriminate among risks. Numerical results are given for seven European BM-systems.

\section{H. Gerber and E. Shiu: Actuarial Bridges to Dynamic Hedging and Option Pricing.}

In this paper, the authors study the pricing of financial options and derivative securities, and their synthetic replications, by means of the primary securities. They show that a time-honored concept in actuarial science-the Esscher transform-is an efficient tool for pricing many options and contingent claims if the logarithm of the prices of the primary securities are certain stochastic processes with stationary and independent increments.

\section{H. Gerber: A Teacher's Remark on Exact Credibility.}

In the classical Bayesian approach to credibility the claims are conditionally independent and identically distributed random variables, with common density $f(x, \theta)$. The unknown parameter $\theta$ is a realisation of a random variable $\Theta$ having initial (prior) density $U(\theta)$. A central question is for which pairs $f(x, \theta)$ and $u(\theta)$ the premium for $X_{t+1}$, given $X_{1}, \ldots, X_{t}$ is a linear expression. The purpose of the paper is to propose an alternative formulation of the general model, from which the classical examples that lead to a linear expression can be retrieved in a straightforward way.

\section{M.D. Dwonczyk, D.E. A. Sanders and G. Kaye: Insurance as a Capital Project.}

The objective of the paper is to consider whether it is possible for an insurer to gain financial advantage by treating his business as being one or more capital projects. Capital project techniques typically involve forecasting the future cash flows arising from the given financial structure. The actuary needs to develop sophisticated financial modelling techniques including the use of a number of suitable and consistent financial assumptions. All risks must be identified, modelled and managed. The paper considers two common structures for insurance suppliers and 
considers the risks and rewards of those structures. The paper then describes the steps involved in a capital project approach to investigating an insurance company.

A. Parisi and S. Martignolles: Utilisation de Modèles sur les Montants Cumulés pour Déterminer la Loi de Probabilité des Provisions pour Sinistres à Payer en Assurance IARD, Part II.

This paper is a continuation of the work performed by the same authors in their paper "Modèles sur les Montants Cumulés pour Déterminer la Loi de Probabilité des Provisions pour Sinistres à Payer en Assurance IARD" which was presented during one of the working sessions on the first topic of this colloquium.

\section{F. Dufresne: An Extension of Kornya's Method with Application to Pension} Funds.

The author considers a simple extension of the method of Kornya for computing aggregate claims in the individual risk theory model. The extended method applies to the convolution of triatomic distributions with nonnegative support. The algorithm can be applied to the calculation of the total claims distribution of a pension fund where only death and disability of active members are considered.

\section{G. Parker and A. Cairns: Pension Fund Modelling with Random Investment Performance.}

This paper considers the stochastic behaviour of the funding level of a defined benefit pension plan through time and its relationship with the plan contribution rate.

First the authors investigate the effect of the valuation basis and of the amortization period on the variability of funding levels and contribution rates and this introduces the concept of the efficient frontier as a means of choosing an optimal funding strategy.

Then they consider models with dependent rates of return and provide a sufficient condition for the funding level to be ergodic. Upon considering the AR (1) model they derive a recursive method for calculating the conditional distribution of the funding level and provide further insight into the main factors which influence the behaviour of the funding level. 